\title{
Distance Constrained Based Adaptive Flocking Control for Multiagent Networks with Time Delay
}

\author{
Qing Zhang, ${ }^{1}$ Ping Li, ${ }^{1}$ Zhengquan Yang, ${ }^{1}$ and Zengqiang Chen ${ }^{1,2}$ \\ ${ }^{1}$ College of Science, Civil Aviation University of China, No. 2898, Jinbei Road, Tianjin 300300, China \\ ${ }^{2}$ Department of Automation, Nankai University, Tianjin 300071, China \\ Correspondence should be addressed to Qing Zhang; qz120168@hotmail.com
}

Received 6 June 2014; Revised 23 September 2014; Accepted 23 September 2014

Academic Editor: He Huang

Copyright ( 2015 Qing Zhang et al. This is an open access article distributed under the Creative Commons Attribution License, which permits unrestricted use, distribution, and reproduction in any medium, provided the original work is properly cited.

\begin{abstract}
The flocking control of multiagent system is a new type of decentralized control method, which has aroused great attention. The paper includes a detailed research in terms of distance constrained based adaptive flocking control for multiagent system with time delay. Firstly, the program on the adaptive flocking with time delay of multiagent is proposed. Secondly, a kind of adaptive controllers and updating laws are presented. According to the Lyapunov stability theory, it is proved that the distance between agents can be larger than a constant during the motion evolution. What is more, velocities of each agent come to the same asymptotically. Finally, the analytical results can be verified by a numerical example.
\end{abstract}

\section{Introduction}

In recent years, the research on the flocking behavior of multiagent system has attracted great attention. For a series of agents which can apply some simple rules and limited information of neighbors to organize into a coordinated state is called flocking phenomenon. There exist many forms of flocking behavior in nature, for example, flocking of birds, swarming of bacteria, and so on $[1,2]$. With the development of technology and the importance of real application, the study of the flocking behaviors of multiagent system has caused attention from a lot of different backgrounds, for example, biology, computer science, physics, and so on [3-8].

There are a plenty of existing works contributing to the flocking problems. Three heuristic rules leading to emergence of the first computer animation of flocking were first reported by Reynolds in 1987 [5]. The essential flocking rules depict how a personal agent maneuvers based on the local flock mates' positions as well as velocities. Vicsek et al. designed a simple flocking model of multiagents which can all move with the same speed but with different directions in the plane. The Vicsek model is a special version of a pattern introduced previously by Reynolds [6]. Jadbabaie et al. put forward the rigorous proof of the convergence for Vicsek model [7]. There are many generalized species of this model, such as a leader follower strategy which means one agent acts as a group leader and the other agents would just follow the leader and keep the cohesion/separation/alignment rules. Recently, various desired state flocking motions are deduced in most cases [9-13]. Zavlanos et al., especially, proposed connectivity preserving controllers, by designing novel interagent potentials, to realize the flocking of multiagent system under the initial connectivity assumption $[12,13]$.

The time delays of systems are a very common phenomenon in real life. Many factors, for example, finite signal transmission speeds and memory effects, can cause time delay in spreading and communication. Therefore, it should be considered to design the control scheme for multiagent system with time delay. The effect of exchange delays for consensus problems and formation problems has been discussed [14-19]. According to the matrix theory and the frequency analysis, $\mathrm{Su}$ et al. obtained the desired moving model with a delay-dependent formation control algorithm [20]. Yang et al. studied the virtual potential approach for stabilising a group of agents at a desired formation [21]. Adaptive control is a kind of very important method in the control of complex nonlinear systems [22-24]. A good adaptive control can adapt to the changes in a large range of parameters of controlled 
system. It can not only maintain stable operation of the system but also keep the optimal in degree. The literatures focus on flocking with collision avoidance. However, the distance between multiagents is required to be larger than a constant $r$ in reality. For example, bird to incite wings must have their own space. Robot teams and UAV (unmanned air vehicles) in the formation movement in order to avoid a collision must consider their size. So, in the flocking control, it is not enough only to require the distance between them to be greater than zero. Motivated by this fact and on the basis of the abovementioned works, distance constrained based adaptive flocking control for multiagent system with time delay is presented in this paper. The innovation of this paper is mainly in the following aspects: the adaptive controller being designed to achieve the adaptive flocking of multiagents with time delays and keeping the distance between multiagents to be larger than a constant $r$. The stability of the adaptive flocking of the multiagent system with time delays is analyzed theoretically. A sufficient condition is given for the stability of the adaptive control system.

The rest of the paper was structured from the following aspects. In Section 2, the multiagent flocking problem and some preliminaries used throughout this paper are introduced. In Section 3, a controller is designed based on adaptive flocking control laws. In Section 4, the main theory results that velocities of each agent come to the same asymptotically and the distance between agents required to be larger than a constant $r$ are proved. In Section 5, a simulation case is also presented to verify the effectiveness of our theoretical results. In Section 6, the full text content is summarized and the further research in the aspects of adaptive flocking of multiagent networks is investigated.

\section{Preliminaries}

A set of $N(N \geq 2)$ agents moving in an $n$-dimensional Euclidean space are considered. The dynamics of each agent is characterized by the following dynamic system (see [25]):

$$
\begin{gathered}
\dot{x}_{i}(t)=v_{i}(t), \\
\dot{v}_{i}(t)=f\left(v_{i}(t)\right)+u_{i}(t), \quad i=1,2, \ldots, N,
\end{gathered}
$$

where $x_{i}(t) \in R_{2}^{n}$ and $v_{i}(t) \in R_{2}^{n}$ are the position vector and the velocity of agent $i$, independently, $f\left(v_{i}(t)\right)$ is a nonlinear vector-valued continuous function which describes the intrinsic dynamics of agent $i$, and $u_{i}(t) \in R_{2}^{n}$ is the control input acting on agent $i$. Especially, the virtual leader for multiagent system (1) is a special agent described by

$$
\dot{x}_{l}(t)=v_{l}(t), \quad \dot{v}_{l}(t)=f\left(v_{l}(t)\right),
$$

where $x_{l}(t) \in R_{2}^{n}$ and $v_{l}(t) \in R_{2}^{n}$ are the position and velocity vector of the virtual leader, respectively. In this paper, an assumption has been made that all agents can get the information of the virtual leader. The information switching between multiagents with the leader exhibits time delays.

Define error vector

$$
e_{i}=v_{i}-v_{l}, \quad i=1,2, \ldots, N
$$

Based on the definition of $e_{i}$, we have the following equations:

$$
\begin{gathered}
v_{j}(t-\tau)-v_{l}(t-\tau)=e_{j}(t-\tau) \quad i, j=1,2, \ldots, N, \\
e_{i}-e_{j}=\left(v_{i}-v_{l}\right)-\left(v_{j}-v_{l}\right)=v_{i}-v_{j} \quad i, j=1,2, \ldots, N .
\end{gathered}
$$

Assumption 1. Assume that there exists a nonnegative constant $\alpha$ satisfying

$$
\left\|f\left(v_{i}\right)-f\left(v_{l}\right)\right\|_{2} \leq \alpha\left\|e_{i}\right\|_{2}, \quad i=1,2, \ldots, N
$$

Definition 2. Communication radius $R_{2}$ is defined as the biggest distance from which multiagents can get information of other agents. Hysteresis radius $R_{1}$ is the distance in which a new edge will not be added to the graph until the distance between any two agents which are not connected decreases to $R_{1}$. Safe radius $r$ is the distance needed by the agent own activities.

Definition 3 (dynamic graphs [10]). We call $G(t)=(V, E(t))$ a dynamic graph which consists of a set of vertices $V=$ $\{1,2, \ldots, N\}$ indexed by the set of agents and a time varying set of links $E(t)=\{(i, j) \mid i, j \in V\}$, such that, for any $0<r<R_{1}<R_{2}$,
(1) if $r<\left\|x_{i}(t)-x_{j}(t)\right\| \leq R_{1}$, then $(i, j) \in E(t)$,
(2) if $R_{2} \leq\left\|x_{i}(t)-x_{j}(t)\right\|$, then $(i, j) \notin E(t)$.

Dynamic graphs $G(t)$ meet the conditions that $(i, j) \in$ $E(t)$ if and only if $(j, i) \in E(t)$ are called undirected, which constitute the key point of the paper. If any vertices $i$ and $j$ in an undirected graph $G(t)$ are joined by a link $(i, j) \in E(t)$, we can call them adjacent or neighbors at time $t$ and denote them by $i \sim j$. Let $L(t)=A(t)-\Delta(t)$ be equivalently represented by a dynamic negative Laplacian matrix, where $A(t)=\left(a_{i j}(t)\right)$ is a weighted adjacency matrix of the graph $G(t)$, which satisfies that $a_{i j}(t)>0$ if $i \sim j, a_{i i}=-\sum_{j=1, j \neq i}^{N} a_{i j}$, and $a_{i j}(t)=0$, otherwise. A topological invariance of graphs, that is, graph connectivity, is of great interest for this paper. The switching process of dynamic graphs can be shown in Figure 1.

Definition 4 (graph connectivity). We say that a dynamic graph $G(t)$ is connected at time $t$ if there exists a path, that is, a sequence of distinct vertices such as the fact that consecutive vertices are adjacent, between any two vertices in $G(t)$.

Hence, the problem which is mentioned can be formally stated as follows.

Definition 5 (flocking). Consider the set of connected graphs $C_{N}$ on $N$ vertices determining the distribution of control laws $u_{i}(t)$ for all agents $i$. If the initial network $G\left(t_{0}\right) \in C_{N}$ and the initial distance of each agent is bigger than $r, G(t) \in C_{N}$ for all time $t \geq t_{0}$, and the distance of multiagents satisfies $\left\|x_{i j}\right\|>r$. Moreover, the velocity of each agent asymptotically approaches the same. 


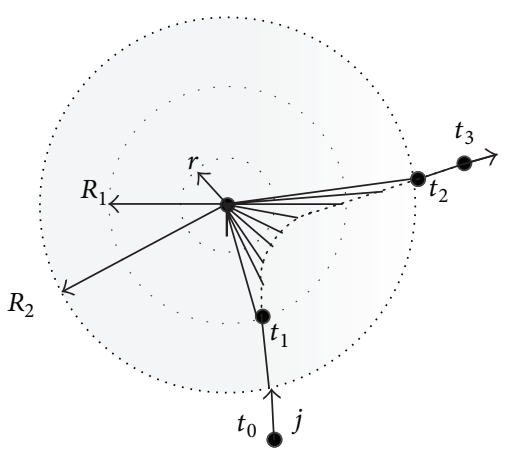

FIGURE 1: The switching process of dynamic graphs according to Definition 3.

\section{The Design for Virtual Leader Based Adaptive Flocking Control Laws}

In view of any dynamic graph $G(t)=(V, E(t))$, we can define the set of control laws,

$$
\begin{gathered}
u_{i}=-2 \sum_{j=1}^{N} a_{i j} \nabla_{x_{i}} V_{i j}-2 \sum_{j=1, j \neq i}^{N} a_{i j}\left(v_{i}(t-\tau)-v_{j}(t-\tau)\right)-d_{i} e_{i}, \\
\dot{d}_{i}=k_{i} e_{i}^{T} e_{i},
\end{gathered}
$$

where $d_{i}$ is the updating parameters, $\left\{k_{i}, i=1,2, \ldots, N\right\}$ are positive constants, $\tau$ is the coupling time delay, and $\nabla_{x_{i}} V_{i j}$ is a direction vector of the negative gradient of an artificial potential function defined by the following equation:

$$
V_{i j}\left(x_{i j}\right)=\frac{1}{R_{2}^{2}-\left\|x_{i j}\right\|^{2}}+\frac{1}{\left\|x_{i j}\right\|^{2}-r^{2}}, \quad\left\|x_{i j}\right\| \in\left(r, R_{2}\right)
$$

with $x_{i j}=x_{i}-x_{j}$, where $V_{i j}$ grows unbound when $\left\|x_{i j}\right\| \rightarrow$ $R_{2}^{-}$, or $\left\|x_{i j}\right\| \rightarrow r^{+}$.

Based on the definition of $V_{i j}$, we have the following equation:

$$
\begin{array}{r}
\nabla_{x_{i j}} V_{i j}\left(x_{i j}\right)=\nabla_{x_{i}} V_{i j}\left(x_{i j}\right)=-\nabla_{x_{j}} V_{i j}\left(x_{i j}\right) \\
i, j=1,2, \ldots, N .
\end{array}
$$

From equations of (4), (7) and $a_{i i}=-\sum_{j=1, j \neq i}^{N} a_{i j}$, it was concluded that

$$
\begin{aligned}
u_{i}= & -2 \sum_{j=1}^{N} a_{i j} \nabla_{x_{i}} V_{i j}-2 \sum_{j=1, j \neq i}^{N} a_{i j}\left(v_{i}(t-\tau)-v_{j}(t-\tau)\right)-d_{i} e_{i} \\
= & -2 \sum_{j=1}^{N} a_{i j} \nabla_{x_{i}} V_{i j}-2 \sum_{j=1, j \neq i}^{N} a_{i j} v_{i}(t-\tau) \\
& +2 \sum_{j=1, j \neq i}^{N} a_{i j} v_{j}(t-\tau)-d_{i} e_{i}
\end{aligned}
$$

$$
\begin{aligned}
& =-2 \sum_{j=1}^{N} a_{i j} \nabla_{x_{i}} V_{i j}+2 a_{i i} v_{i}(t-\tau)+2 \sum_{j=1, j \neq i}^{N} a_{i j} v_{j}(t-\tau)-d_{i} e_{i} \\
& =-2 \sum_{j=1}^{N} a_{i j} \nabla_{x_{i}} V_{i j}+2 \sum_{j=1}^{N} a_{i j} v_{j}(t-\tau)-d_{i} e_{i} \\
& =-2 \sum_{j=1}^{N} a_{i j} \nabla_{x_{i}} V_{i j}+2 \sum_{j=1}^{N} a_{i j} v_{j}(t-\tau)-2 \sum_{j=1}^{N} a_{i j} v_{l}(t-\tau)-d_{i} e_{i} \\
& =-2 \sum_{j=1}^{N} a_{i j} \nabla_{x_{i}} V_{i j}+2 \sum_{j=1}^{N} a_{i j} e_{j}(t-\tau)-d_{i} e_{i} \\
& i=1,2, \ldots, N .
\end{aligned}
$$

From (1), (2), and (10), we can get the error equation

$$
\begin{array}{r}
\dot{e}_{i}=f\left(v_{i}\right)-f\left(v_{l}\right)-2 \sum_{j=1}^{N} a_{i j} \nabla_{x_{i}} V_{i j}+2 \sum_{j=1}^{N} a_{i j} e_{j}(t-\tau)-d_{i} e_{i} \\
i=1,2, \ldots, N .
\end{array}
$$

We will give some main results for our proposed scheme in next chapter.

\section{The Main Theory Results}

For the considered multiagent system, we can assume that $G(t)$ switches at time $t_{p}$ for $p=1,2, \ldots$. That is to say, $G(t)$ is a fixed graph in each time interval $\left[t_{p-1}, t_{p}\right)$. At the same time, we can define a switching signal $g(t):\left[t_{0}, \infty\right) \rightarrow$ $C_{N}$ associated with connected graphs, and we can have the following conclusion.

Theorem 6. For the multiagent systems (1) and (2), the switching signal $g(t)$ satisfies $E\left(t_{p}\right) \subseteq E\left(t_{q}\right)$ for any pair of switching times $t_{p} \leq t_{q}$ under the control laws (10).

Proof. Consider the following semipositive definite function:

$$
\begin{aligned}
V_{G}= & \frac{1}{2} \sum_{i=1}^{N} e_{i}^{T} e_{i}+\frac{1}{2} \sum_{i=1}^{N} \frac{\left(d_{i}-\widehat{d}_{i}\right)^{2}}{k_{i}}+\sum_{i=1}^{N} \sum_{j=1}^{N} a_{i j} V_{i j} \\
& +\sum_{i=1}^{N} \int_{-\tau}^{0} e_{i}^{T}(t+\eta) e_{i}(t+\eta) d \eta,
\end{aligned}
$$

where $\widehat{d}_{i}$ is a sufficiently large positive constant.

The generalized time derivative of $V_{G}$ is

$$
\begin{aligned}
\dot{V}_{G}= & \sum_{i=1}^{N} e_{i}^{T} \dot{e}_{i}+\sum_{i=1}^{N} \frac{\left(d_{i}-\widehat{d}_{i}\right)}{k_{i}} \dot{d}_{i}+\sum_{i=1}^{N} \sum_{j=1}^{N} a_{i j} \dot{V}_{i j} \\
& +\sum_{i=1}^{N}\left[e_{i}^{T} e_{i}-e_{i}^{T}(t-\tau) e_{i}(t-\tau)\right] .
\end{aligned}
$$


Due to (9), we have

$$
\begin{aligned}
\sum_{i=1}^{N} \sum_{j=1}^{N} a_{i j} \dot{V}_{i j} & =\sum_{i=1}^{N} \sum_{j=1}^{N} a_{i j} \dot{x}_{i j}^{T} \nabla_{x_{i j}} V_{i j} \\
& =\sum_{i=1}^{N} \sum_{j=1}^{N} a_{i j}\left(\dot{x}_{i}^{T} \nabla_{x_{i j}} V_{i j}-\dot{x}_{j}^{T} \nabla_{x_{i j}} V_{i j}\right) \\
& =\sum_{i=1}^{N} \sum_{j=1}^{N} a_{i j}\left(\dot{x}_{i}^{T} \nabla_{x_{i}} V_{i j}+\dot{x}_{j}^{T} \nabla_{x_{j}} V_{i j}\right) \\
& =\sum_{i=1}^{N} \sum_{j=1}^{N} a_{i j}\left(v_{i}^{T} \nabla_{x_{i}} V_{i j}+v_{j}^{T} \nabla_{x_{j}} V_{i j}\right) \\
& =\sum_{i=1}^{N} \sum_{j=1}^{N} a_{i j}\left(v_{i}^{T}-v_{j}^{T}\right) \nabla_{x_{i}} V_{i j} .
\end{aligned}
$$

Then, from (5), we have

$$
\begin{aligned}
& \sum_{i=1}^{N} \sum_{j=1}^{N} a_{i j}\left(v_{i}^{T}-v_{j}^{T}\right) \nabla_{x_{i}} V_{i j} \\
& =\sum_{i=1}^{N} \sum_{j=1}^{N} a_{i j}\left(e_{i}^{T}-e_{j}^{T}\right) \nabla_{x_{i}} V_{i j} \\
& =\sum_{i=1}^{N} \sum_{j=1}^{N}\left(a_{i j} e_{i}^{T} \nabla_{x_{i}} V_{i j}+a_{i j} e_{j}^{T} \nabla_{x_{j}} V_{i j}\right) \\
& =2 \sum_{i=1}^{N} \sum_{j=1}^{N} a_{i j} e_{i}^{T} \nabla_{x_{i}} V_{i j} .
\end{aligned}
$$

The first two parts of (13) can be rewritten as the following forms:

$$
\begin{aligned}
\sum_{i=1}^{N} e_{i}^{T} \dot{e}_{i}+\sum_{i=1}^{N} \frac{\left(d_{i}-\widehat{d}_{i}\right)}{k_{i}} \dot{d}_{i} & \\
= & \sum_{i=1}^{N} e_{i}^{T}\left(f\left(v_{i}\right)-f\left(v_{l}\right)-2 \sum_{j=1}^{N} a_{i j} \nabla_{x_{i}} V_{i j}\right. \\
& \left.\quad+2 \sum_{j=1}^{N} a_{i j} e_{j}(t-\tau)-d_{i} e_{i}\right)+\sum_{i=1}^{N}\left(d_{i}-\widehat{d}_{i}\right) e_{i}^{T} e_{i} \\
= & \sum_{i=1}^{N} e_{i}^{T}\left(f\left(v_{i}\right)-f\left(v_{l}\right)\right)-2 \sum_{i=1}^{N} \sum_{j=1}^{N} a_{i j} e_{i}^{T} \nabla_{x_{i}} V_{i j} \\
& +2 \sum_{i=1}^{N} \sum_{j=1}^{N} a_{i j} e_{i}^{T} e_{j}(t-\tau)-\sum_{i=1}^{N} d_{i} e_{i}^{T} e_{i}+\sum_{i=1}^{N}\left(d_{i}-\widehat{d}_{i}\right) e_{i}^{T} e_{i} \\
= & \sum_{i=1}^{N} e_{i}^{T}\left(f\left(v_{i}\right)-f\left(v_{l}\right)\right)-2 \sum_{i=1}^{N} \sum_{j=1}^{N} a_{i j} e_{i}^{T} \nabla_{x_{i}} V_{i j} \\
& +2 \sum_{i=1}^{N} \sum_{j=1}^{N} a_{i j} e_{i}^{T} e_{j}(t-\tau)-\sum_{i=1}^{N} e_{i}^{T} \widehat{d}_{i} e_{i}
\end{aligned}
$$

$$
\begin{aligned}
= & -2 \sum_{i=1}^{N} \sum_{j=1}^{N} a_{i j} e_{i}^{T} \nabla_{x_{i}} V_{i j}+2 \sum_{i=1}^{N} \sum_{j=1}^{N} a_{i j} e_{i}^{T} e_{j}(t-\tau) \\
& +\sum_{i=1}^{N} e_{i}^{T}\left(f\left(v_{i}\right)-f\left(v_{l}\right)-\widehat{d}_{i} e_{i}\right) \\
\leq & -2 \sum_{i=1}^{N} \sum_{j=1}^{N} a_{i j} e_{i}^{T} \nabla_{x_{i}} V_{i j}+2 \sum_{i=1}^{N} \sum_{j=1}^{N} a_{i j} e_{i}^{T} e_{j}(t-\tau) \\
& +\sum_{i=1}^{N}\left(\alpha-\widehat{d}_{i}\right) e_{i}^{T} e_{i} .
\end{aligned}
$$

Thus, we can obtain

$$
\begin{aligned}
\dot{V}_{G} \leq & -2 \sum_{i=1}^{N} \sum_{j=1}^{N} a_{i j} e_{i}^{T} \nabla_{x_{i}} V_{i j}+2 \sum_{i=1}^{N} \sum_{j=1}^{N} a_{i j} e_{i}^{T} e_{j}(t-\tau) \\
& +\sum_{i=1}^{N}\left(\alpha-\widehat{d}_{i}\right) e_{i}^{T} e_{i}+2 \sum_{i=1}^{N} \sum_{j=1}^{N} a_{i j} e_{i}^{T} \nabla_{x_{i}} V_{i j} \\
& +\sum_{i=1}^{N}\left[e_{i}^{T} e_{i}-e_{i}^{T}(t-\tau) e_{i}(t-\tau)\right]=2 \sum_{i=1}^{N} \sum_{j=1}^{N} a_{i j} e_{i}^{T} e_{j}(t-\tau) \\
& +\sum_{i=1}^{N}\left[e_{i}^{T} e_{i}-e_{i}^{T}(t-\tau) e_{i}(t-\tau)\right]+\sum_{i=1}^{N}\left(\alpha-\widehat{d}_{i}\right) e_{i}^{T} e_{i} \\
\leq & 2 \sum_{i=1}^{N} \sum_{j=1}^{N}\left|a_{i j}\right|\left\|e_{i}^{T}\right\|\left\|e_{j}(t-\tau)\right\|-\sum_{i=1}^{N} e_{i}^{T}(t-\tau) e_{i}(t-\tau) \\
& +\sum_{i=1}^{N} e_{i}^{T} e_{i}+\sum_{i=1}^{N}\left(\alpha-\widehat{d_{i}}\right) e_{i}^{T} e_{i} \\
= & 2 \mathbf{e}^{T} \Gamma \mathbf{e}(t-\tau)-\mathbf{e}(t-\tau)^{T} \mathbf{e}(t-\tau)+\mathbf{e}^{T} \Lambda \mathbf{e} \\
\leq & \mathbf{e}^{T} \Gamma^{T} \Gamma \mathbf{e}+\mathbf{e}^{T} \Lambda \mathbf{e}=\mathbf{e}^{T}\left(\Gamma^{T} \Gamma+\Lambda\right) \mathbf{e}
\end{aligned}
$$

where

$$
\begin{gathered}
\mathbf{e}=\left(\left\|e_{1}\right\|_{2},\left\|e_{2}\right\|_{2}, \ldots,\left\|e_{N}\right\|_{2}\right)^{T}, \\
\mathbf{e}(t-\tau)=\left(\left\|e_{1}(t-\tau)\right\|_{2},\left\|e_{2}(t-\tau)\right\|_{2}, \ldots,\left\|e_{N}(t-\tau)\right\|_{2}\right)^{T}, \\
\Gamma=\left(\left|a_{i j}\right|\right)_{N \times N}, \\
\Lambda=\left(\begin{array}{ccc}
\alpha-\widehat{d}_{1}+1 & 0 \\
0 & \ddots & \alpha-\widehat{d}_{N}+1
\end{array}\right) .
\end{gathered}
$$

As $\alpha$ and $\left|a_{i j}\right|$ are nonnegative, one can select suitable positive constants $\widehat{d}_{i}(1 \leq i \leq N)$ to make

$$
\left(\Lambda+\Gamma^{T} \Gamma\right) \leq 0 .
$$


So, it follows that $\dot{V}_{G} \leq 0$. Hence, $\dot{V}_{G}$ is negative semidefinite for any signal $g(t)$.

Because $\dot{V}_{G}$ is negative semidefinite, $V_{G}$ cannot increase for all time. In addition, the $V_{G}$ is bounded. In other words, it means that, for any $(i, j) \in E(t), V_{i j}$ is bounded. On the one hand, if for some $(i, j) \in E(t),\left\|x_{i j}\right\| \rightarrow R_{2}, V_{i j}\left(x_{i j}\right) \rightarrow \infty$. Thus, by continuity of $V_{G}$, it follows that $\left\|x_{i j}\right\|<R_{2}$, for all $(i, j) \in E(t)$ and $t \in\left[t_{p}, t_{p+1}\right]$. In other words, all links in $V_{G}$ are preserved between switching times, which implies that $E\left(t_{p}\right) \subseteq E\left(t_{q}\right)$. Apply this recursive argument to complete the proof. A similar argument for the case where $x_{i j} \rightarrow r^{+}$can be used to establish that the distance of multiagents satisfies $\left\|x_{i j}\right\|>r$ for all time $t \geq t_{0}$.

Clearly, Theorem 6 explains that the switching signal will satisfy $G(t) \in C_{N}$ for all time $t>t_{0}$, if $G\left(t_{0}\right) \in C_{N}$. We, specially, have the following corollary.

Corollary 7. Under control law (7), the total number of switching times of system (1) is finite.

Proof. The size of the set of links $E\left(t_{0}\right)$ forms an increasing sequence and

$$
\sup _{t \geq t_{0}}|E(t)|-\left|E\left(t_{0}\right)\right|=\frac{n(n-1)}{2}-(n-1),
$$

where $n-1$ is the number of links in $G\left(t_{0}\right)$ if it is minimally connected, that is, if it is a tree, and $n(n-1) / 2$ corresponds to the number of links in a complete graph.

Theorem 8. Take a group of $N$ agents with dynamics (1), (2), each steered by protocol (10) into account. Suppose that initial network $G(t)$ is connected and the initial distance of each agent is bigger than $r$. Then, $G(t)$ is connected for all $t \geq 0$, the velocity of each agent asymptotically approaches the same, and the distance of each agent is bigger than $r$ for all $t \geq 0$.

Proof. The number of switching times of the closed loop system is finite by Corollary 7. And by Theorem 6 if $G\left(t_{0}\right)$ is connected, we can draw the conclusion that $G(t)$ is connected for all time $t>t_{0}$ and so eventually $G(t) \rightarrow G \in C_{N}$.

Referring to the analysis method of [22-24], we will give the proof of the conclusions that the velocity of each agent asymptotically approaches the same and the distance of each agent is bigger than $r$ for all $t \geq 0$.

From (17) and (19), we know that $V_{G}$ is monotonically decreasing and having a lower bound. So, it concludes that $V_{G}$ asymptotically converges to a finite nonnegative value. And, from (17), we have $\dot{V}_{G} \leq \mathrm{e}^{T}\left(\Lambda+\Gamma^{T} \Gamma\right) \mathbf{e}$. When $\widehat{d}_{i}, i=$ $1,2, \ldots, N$ are sufficiently large positive constants, we can see that there exists a nonnegative constant $\lambda$ satisfying

$$
\dot{V}_{G} \leq-\lambda \mathbf{e}^{T} \mathbf{e} .
$$

Thus, we have

$$
\|\mathbf{e}\|^{2} \leq-\frac{\dot{V}_{G}}{\lambda} .
$$

Because $\lim _{t \rightarrow+\infty} \int_{0}^{t}-\left(\dot{V}_{G} / \lambda\right) d \eta$ exists, we can derive from (22) that $\lim _{t \rightarrow+\infty} \int_{0}^{t}\|\mathbf{e}\|^{2} d \eta$ exists by the generalized integral principle.

We can define

$$
\begin{aligned}
V_{G 1} & =\frac{1}{2} \sum_{i=1}^{N} \frac{\left(d_{i}-\widehat{d}_{i}\right)^{2}}{k_{i}}, \\
V_{G 2} & =\sum_{i=1}^{N} \sum_{j=1}^{N} a_{i j} V_{i j}, \\
V_{G 3} & =\sum_{i=1}^{N} \int_{-\tau}^{0} e_{i}^{T}(t+\eta) e_{i}(t+\eta) d \eta .
\end{aligned}
$$

Then, we can get the following equation:

$$
V_{\mathrm{G}}=\frac{1}{2} \sum_{i=1}^{N} e_{i}^{T} e_{i}+V_{\mathrm{G} 1}+V_{\mathrm{G} 2}+V_{\mathrm{G} 3} .
$$

The generalized time derivative of $V_{G 1}$ is $\dot{V}_{G 1}=\sum_{i=1}^{N}\left(d_{i}-\right.$ $\left.\widehat{d}_{i}\right) e_{i}^{T} e_{i}$. It is negative when $\left\{\widehat{d}_{i}, i=1,2, \ldots, N\right\}$ are sufficiently large positive constants. We can conclude that $V_{G 1}$ asymptotically converges to a finite nonnegative value. On the other hand, $V_{G 2}$ asymptotically converges to a finite nonnegative value based on the definition of $V_{i j}$ (refer to (8)). At the same time, the generalized time derivative of $V_{G 3}$ is $\dot{V}_{G 3}=\sum_{i=1}^{N}\left[e_{i}^{T} e_{i}-e_{i}^{T}(t-\tau) e_{i}(t-\tau)\right]$ which asymptotically converges to zero. Therefore, we can derive that $V_{G 3}$ asymptotically converges nonnegative value. By the boundedness of $V_{G}$ and (24), therefore, we can derive that $\lim _{t \rightarrow+\infty} \sum_{i=1}^{N} e_{i}^{T} e_{i}$ exists and is a nonnegative real number. In what follows, we will prove that

$$
\lim _{t \rightarrow+\infty} \sum_{i=1}^{N} e_{i}^{T} e_{i}=0 .
$$

If this is not true, we have

$$
\lim _{t \rightarrow+\infty} \sum_{i=1}^{N} e_{i}^{T} e_{i}=\mu_{1}>0
$$

Then, there obviously exists a real number $M>0$ and $0<\mu<\mu_{1}$, such that $\sum_{i=1}^{N} e_{i}^{T} e_{i}>\mu>0$ for $t \geq M$. From (21), we can get

$$
\dot{V}_{G} \leq-\lambda \sum_{i=1}^{N} e_{i}^{T} e_{i}<-\lambda \mu, \quad t \geq M .
$$

By integrating (25) with respect to $t$ over the time period $M$ to $+\infty$, we can obtain

$$
-V_{G}(M) \leq V_{G}(+\infty)-V_{G}(M)
$$

$$
=\int_{M}^{+\infty} \dot{V}_{G}(t) d t<-\int_{M}^{+\infty} \lambda \mu d t=-\infty .
$$


This yields a contradiction, and so

$$
\lim _{t \rightarrow+\infty} \sum_{i=1}^{N} e_{i}^{T} e_{i}=0
$$

which implies that $\lim _{t \rightarrow+\infty} e_{i}=0$. Moreover, it is natural to see $\lim _{t \rightarrow+\infty} v_{i}=v_{l}$. Thus, in the steady state, all agent velocities asymptotically become the same. Furthermore, in the steady state, since $e_{i}=0$ for $i=1,2, \ldots, N$, we can get

$$
\frac{d}{d t}\left\|x_{i}-x_{j}\right\|^{2}=2\left(x_{i}-x_{j}\right)^{T}\left(v_{i}-v_{j}\right)=0,
$$

and so the distances between agents are invariant.

Finally, we prove that the distance of each gent is bigger than $r$ for all $t \geq 0$. From above proof, we have that $V_{G}$ is bounded for all $t \geq t_{0}$. Also from the definite of potential function, if $x_{i j} \rightarrow r$, we can get that $V_{G}$ is unbounded. Therefore, the distance of each agent is bigger than $r$ for all $t \geq 0$.

\section{Simulation Results}

In this section, an example was presented to show the effectiveness of our proposed algorithm. The potential function $V_{i j}$ is defined in (8) with $R_{2}=5, r=1$, and with hysteresis $R_{1}=4.5$. The simulation is performed with 20 agents in $R_{2}^{2}$. Each agent's initial velocities, labeled with dots, are randomly selected in the unit square. The initial positions distributed on the perimeter of a circle of radius $d=1.5$ on average (Figure 2). In particular, we assume the velocity is $v_{0}(t)=$ $[\sin (t), \cot (t)]^{T}$ and send a signal to one agent which is selected randomly in this simulation. Solid curves connecting every agent indicate the vicinal traveled paths, while arrows indicate the agents velocities. The networks connectivity is guaranteed for all time and the groups asymptotic flocking is achieved, where $\tau=0.5$. Figure 2 is the initial state. Figure 3 indicates the final steady state configuration. Figure 4 is the velocity error plot which is used for describing the errors between agent actual velocities, where the velocity error curves were indicated by the solid lines, and it demonstrates that all agent velocities asymptotically tend to the same. Figure 5 is changing curve of control laws. Figure 6 is the distance $\left\|x_{i j}\right\|$ between agent $i$ and agent $j$ and agent $i, j \in$ $N(t)$. From which one can see that the distance between all agents is larger than $r=1$ for all $t \geq 0$. This simulation results have well verified the theory results.

\section{Conclusions}

In this paper, the adaptive flocking of multiagents with time delay is studied. A novel adaptive flocking control method for multiagents is proposed, and the control law is designed depending on functions of the state information and the external signal. By the control law, all agents can follow the virtual leader and can ensure freedom from collisions between neighboring agents. Some theoretical results are
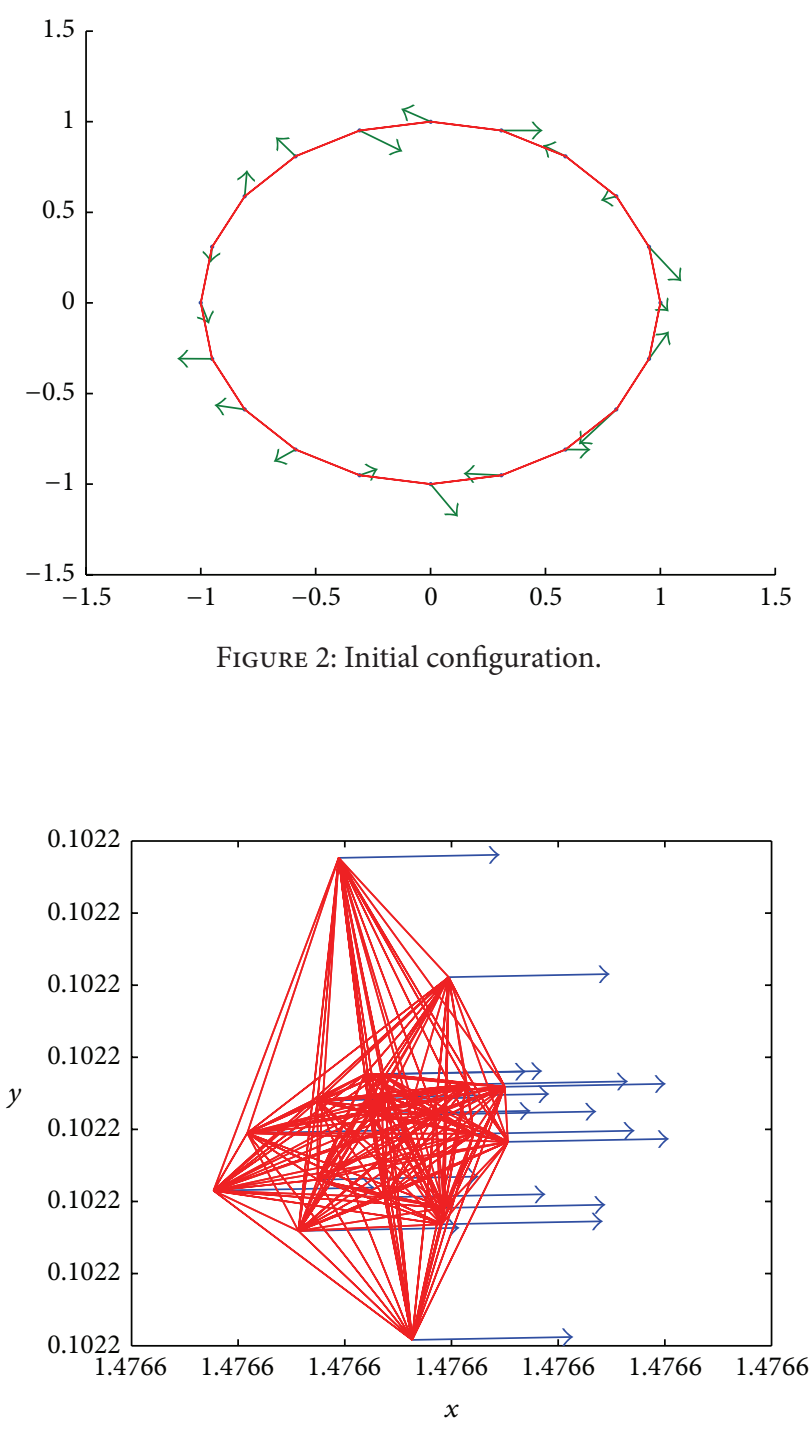

FIGURE 3: End configuration.

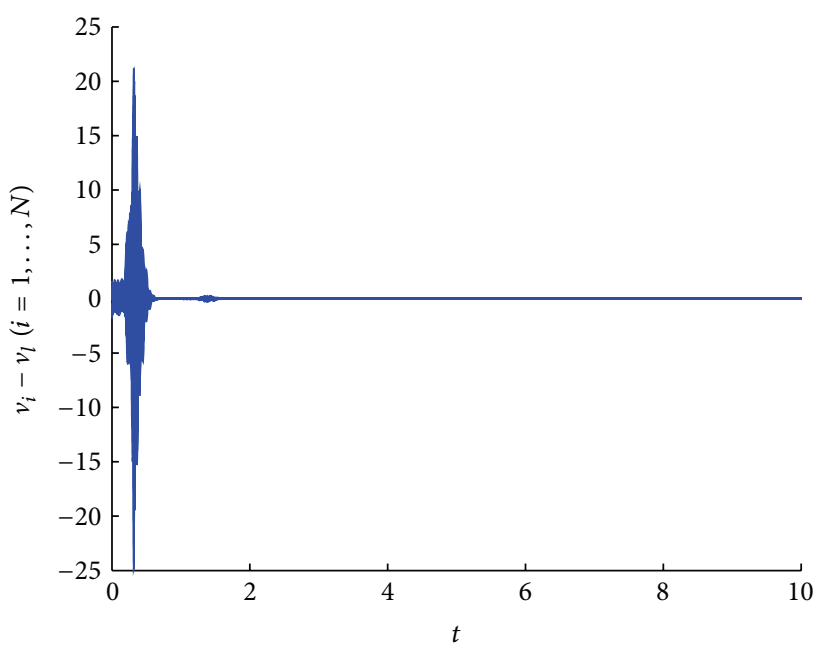

Figure 4: Velocity errors. 


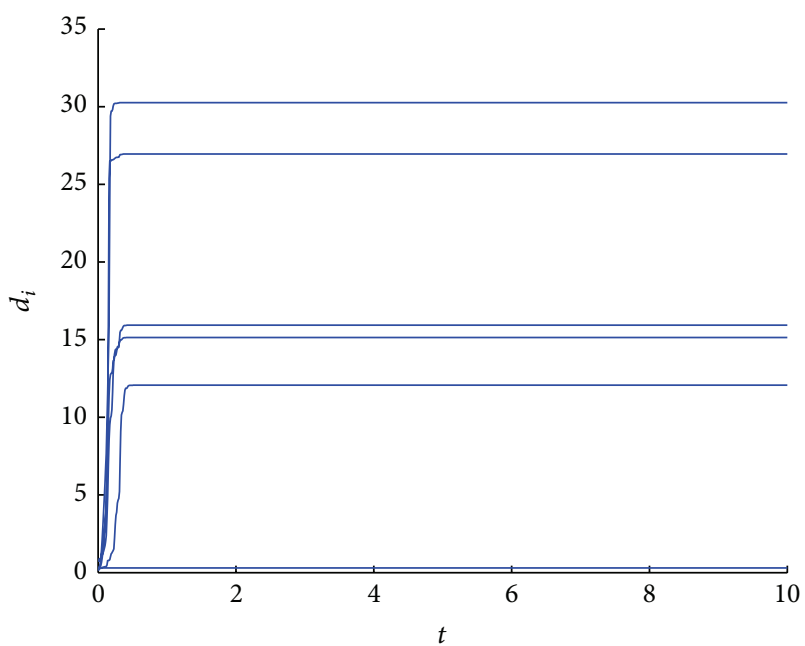

FIgURE 5: Changing curve of control laws.

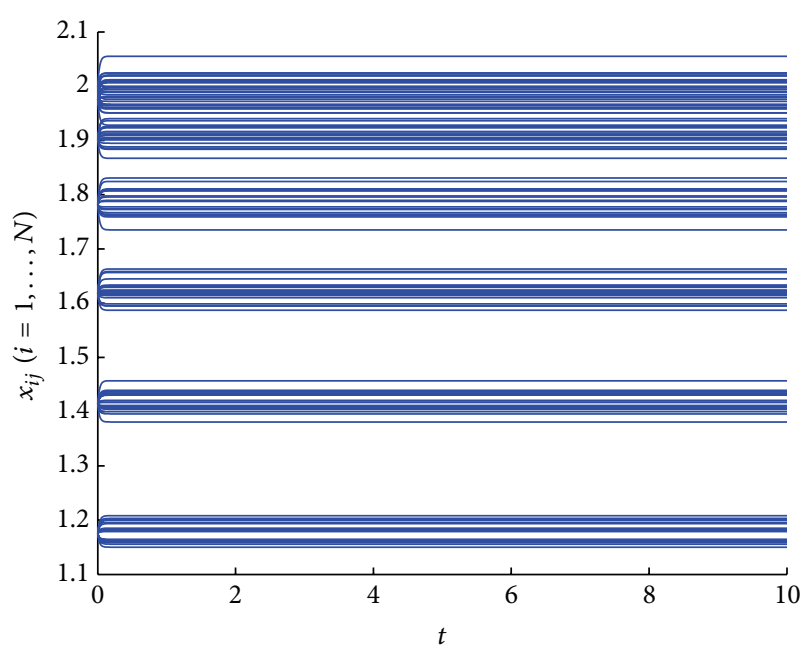

FIGURE 6: Distance of multiagents.

attained, and a numerical example is given to show the practicability of the proposed method. The distance constrained based adaptive flocking control for multiagent system with time delay is proposed in this paper, which has received the expected results. But there are still some problems to be resolved in future research. Firstly, the research of the distance constrained based adaptive flocking control for multiagent system with time delay is achieved on the basics of the strongly connected network. We can study the adaptive flocking control for multiagent system based on the networks which contains a directed spanning tree. Secondly, we can apply the algorithm proposed in this paper to the concrete platform of multiple mobile robots or wireless sensor network, which can combine theory with practice better.

\section{Conflict of Interests}

The authors declare that there is no conflict of interests regarding the publication of this paper.

\section{Acknowledgments}

This work is supported by National Natural Science Foundation of China (61174094, 61039001), National Natural Science Foundation of Tianjin (14JCYBJC18700), Technology and Innovation Fund Project of Civil Aviation University Of China (SY-1415), and Basic Research Projects of High Education (ZXH2010D011, ZXH2012 K002).

\section{References}

[1] E. Shaw, "Fish in schools," Nature History, vol. 84, no. 8, pp. 4045, 1975.

[2] M. S. Tang, X. J. Mao, and Z. Guessoum, "Research on an infectious disease transmission by flocking birds," The Scientific World Journal, vol. 2013, Article ID 196823, 7 pages, 2013.

[3] K. D. Do, "Flocking for multiple elliptical agents with limited communication ranges," IEEE Transactions on Robotics, vol. 27, no. 5, pp. 931-942, 2011.

[4] A. Ferrara and C. Vecchio, "Collision avoidance strategies and coordinated control of passenger vehicles," Nonlinear Dynamics, vol. 49, no. 4, pp. 475-492, 2007.

[5] C. W. Reynolds, "Flocks, herds, and schools: a distributed behavioral model," in Proceedings of the 14th Annual Conference on Computer Graphics and Interactive Techniques, (SIGGRAPH '87), vol. 21, pp. 25-34, ACM, 1987.

[6] T. Vicsek, A. Czirók, E. Ben-Jacob, I. Cohen, and O. Shochet, "Novel type of phase transition in a system of self-driven particles," Physical Review Letters, vol. 75, no. 6, pp. 1226-1229, 1995.

[7] A. Jadbabaie, J. Lin, and A. S. Morse, "Coordination of groups of mobile autonomous agents using nearest neighbor rules," IEEE Transactions on Automatic Control, vol. 48, no. 6, pp. 988-1001, 2003.

[8] J. Toner, Y. Tu, and S. Ramaswamy, "Hydrodynamics and phases of flocks," Annals of Physics, vol. 318, no. 1, pp. 170-244, 2005.

[9] R. Olfati-Saber, "Flocking for multi-agent dynamic systems: algorithms and theory," IEEE Transactions on Automatic Control, vol. 51, no. 3, pp. 401-420, 2006.

[10] H. G. Tanner, A. Jadbabaie, and G. J. Pappas, "Stable flocking of mobile agents, part I: fixed topology," in Proceedings of the 42nd IEEE Conference on Decision and Control, pp. 2010-2015, Maui, Hawaii, USA, December 2003.

[11] H. G. Tanner, A. Jadbabaie, and G. J. Pappas, "Stable flocking of mobile agents, part II: dynamic topology," in Proceedings of the IEEE Conference on Decision and Control, pp. 2016-2021, Maui, Hawaii, USA, 2003.

[12] M. M. Zavlanos, A. Jadbabaie, and G. J. Pappas, "Flocking while preserving network connectivity," in Proceedings of the 46th IEEE Conference on Decision and Control (CDC '07), pp. 29192924, New Orleans, La, USA, December 2007.

[13] H. G. Tanner, A. Jadbabaie, and G. J. Pappas, "Flocking in fixed and switching networks," IEEE Transactions on Automatic Control, vol. 52, no. 5, pp. 863-868, 2007.

[14] L. Moreau, "Stability of continuous-time distributed consensus algorithms," Tech. Rep., School of Electronics and Computer Science, University of Southampton, 2004, http://arxiv.org/pdf/ math/0306426.pdf.

[15] R. Olfati-Saber and R. M. Murray, "Consensus problems in networks of agents with switching topology and time-delays," IEEE Transactions on Automatic Control, vol. 49, no. 9, pp. 15201533, 2004. 
[16] J. P. Hu and Y. G. Hong, "Leader-following coordination of multi-agent systems with coupling time delays," Physica A, vol. 374, no. 2, pp. 853-863, 2007.

[17] F. Xiao and L. Wang, "State consensus for multi-agent systems with switching topologies and time-varying delays," International Journal of Control, vol. 79, no. 10, pp. 1277-1284, 2006.

[18] C.-L. Liu and Y.-P. Tian, "Formation control of multi-agent systems with heterogeneous communication delays," International Journal of Systems Science, vol. 40, no. 6, pp. 627-636, 2009.

[19] S. Ristian and F. Cesare, "Formation control over delayed communication networks," in Proceedings of the IEEE International Conference on Robotics and Automation, pp. 536-538, Pasadena, Calif, USA, May 2008.

[20] H. Su, N. Zhang, M. Z. Chen, H. Wang, and X. Wang, "Adaptive flocking with a virtual leader of multiple agents governed by locally Lipschitz nonlinearity," Nonlinear Analysis: Real World Applications, vol. 14, no. 1, pp. 798-806, 2013.

[21] Z. Yang, Q. Zhang, Z. Jiang, and Z. Chen, "Flocking of multiagents with time delay," International Journal of Systems Science, vol. 43, no. 11, pp. 2125-2134, 2012.

[22] J.-L. Wang, H.-N. Wu, and L. Guo, "Novel adaptive strategies for synchronization of linearly coupled neural networks with reaction-diffusion terms," IEEE Transactions on Neural Networks and Learning Systems, vol. 25, no. 2, pp. 429-440, 2014.

[23] J. L. Wang and H. N. Wu, "Synchronization and adaptive control of an array of linearly coupled reaction-diffusion neural networks with hybrid coupling," IEEE Transactions on Cybernetics, vol. 44, no. 8, pp. 1350-1361, 2014.

[24] J.-L. Wang and H.-N. Wu, "Adaptive output synchronization of complex delayed dynamical networks with output coupling," Neurocomputing, vol. 142, pp. 174-181, 2014.

[25] J.-L. Wang and H.-N. Wu, "Leader-following formation control of multi-agent systems under fixed and switching topologies," International Journal of Control, vol. 85, no. 6, pp. 695-705, 2012. 


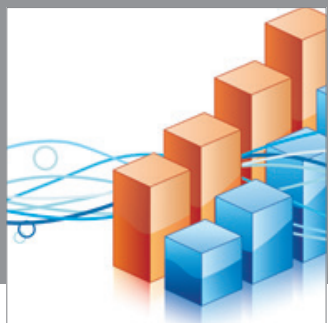

Advances in

Operations Research

mansans

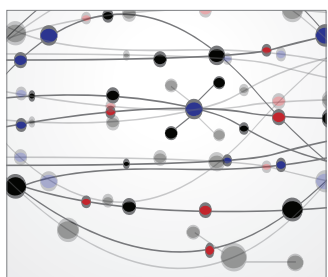

The Scientific World Journal
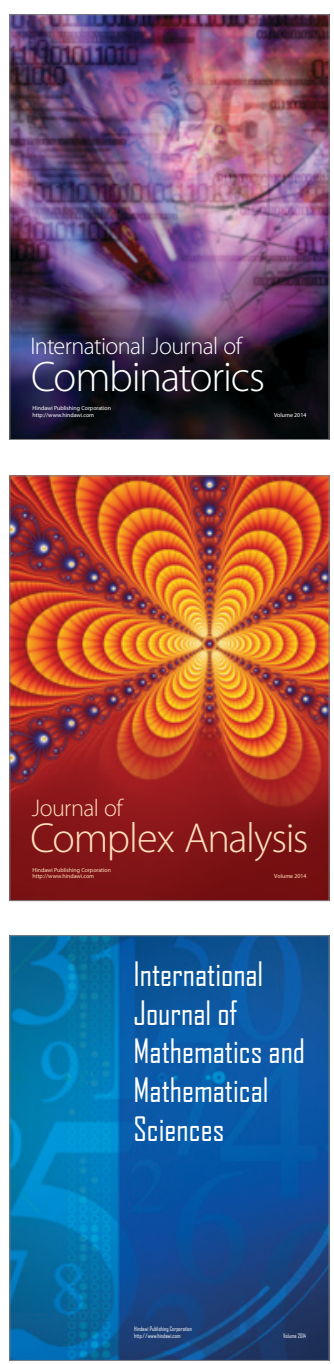
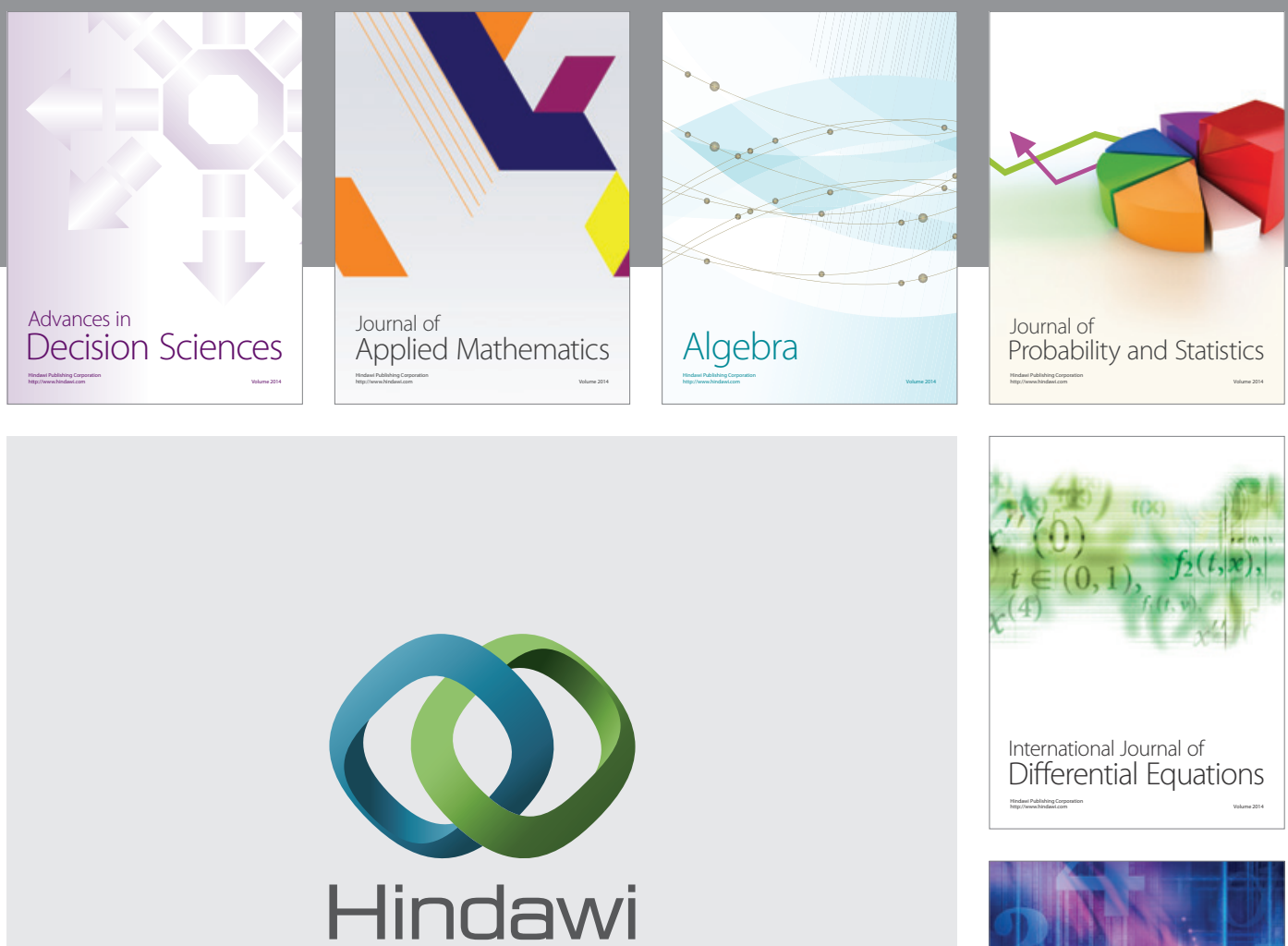

Submit your manuscripts at http://www.hindawi.com
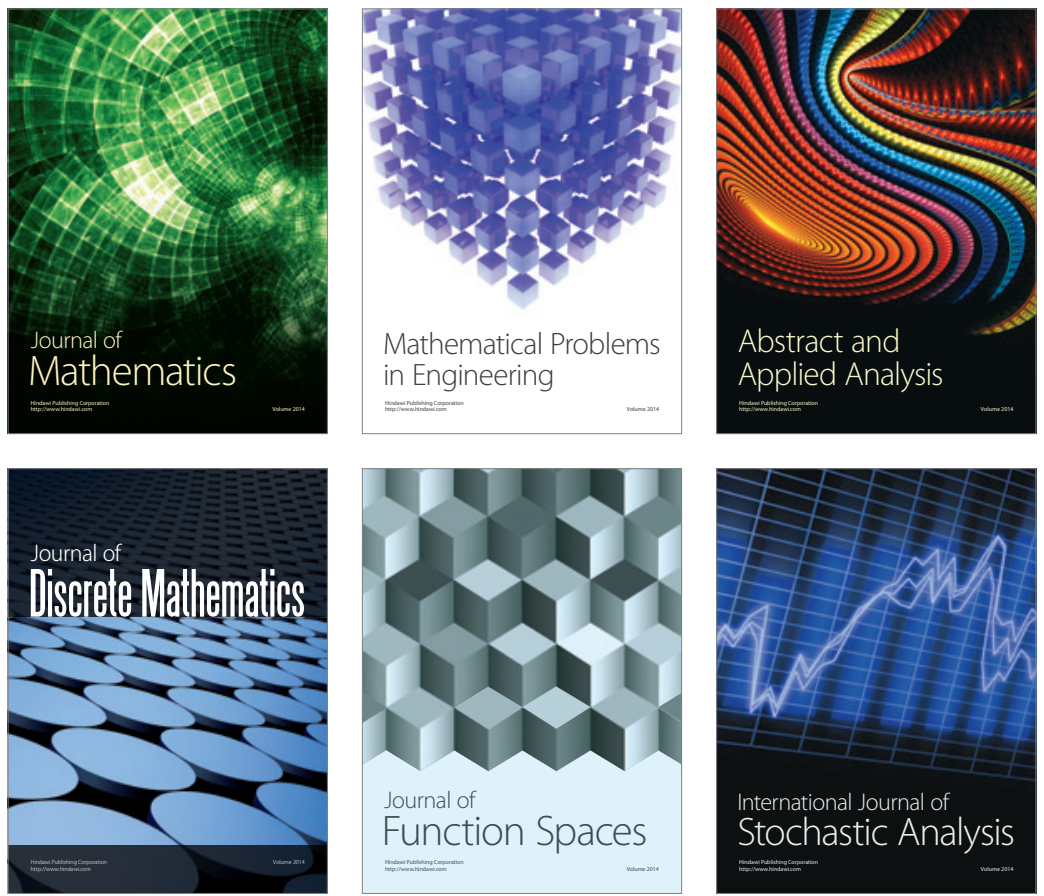

Journal of

Function Spaces

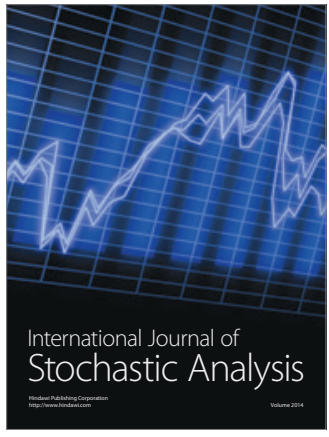

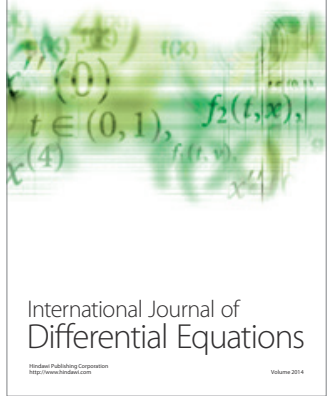
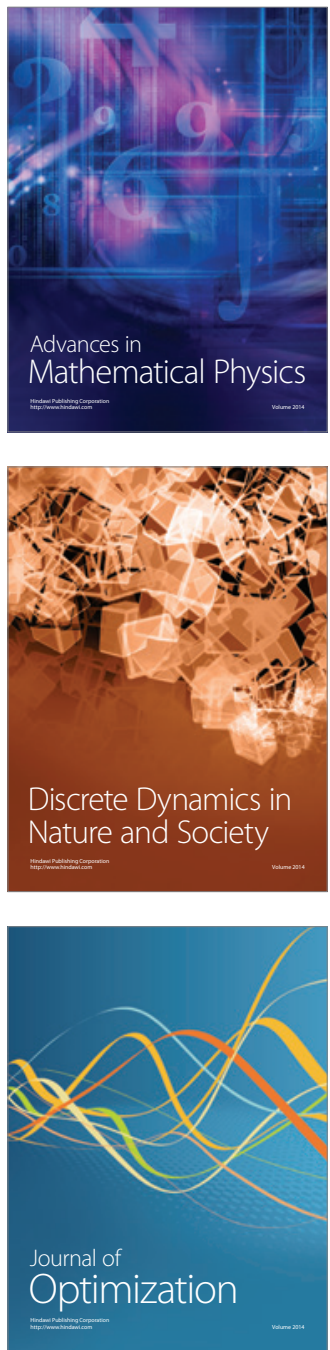